\title{
Editorial
}

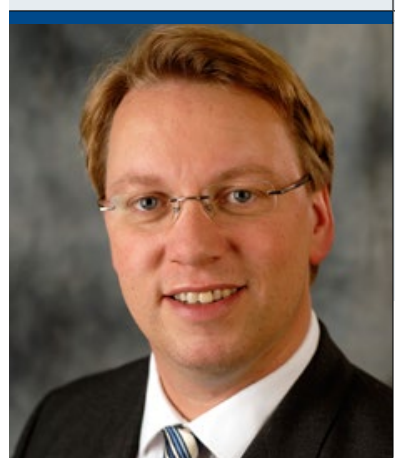

"Mit vergleichsweise wenigen Informationen könn(t)en wir

Urologen unsere Patienten mit einem Harnblasenkarzinom

risikoprofiladaptiert diagnostizieren, therapieren und sogar

nachsorgen."

\section{Keep it simple, stupid!}

$\mathrm{K}$ eep it simple, stupid (KISS)! Halte es einfach, Dummkopf! Nein, liebe Kolleginnen und Kollegen, dass nehmen Sie jetzt bitte nicht wörtlich. Mach's so einfach wie möglich - schon besser und nebenbei ganz schnell bei Wikipedia nachgeschaut.

\section{Der erste Einsatz als Beirat}

Als neues Beiratsmitglied der URO-NEWS möchte ich an dieser Stelle meine Vorliebe für die angelsächsische Betrachtung zeitgenössischer Probleme im Allgemeinen und medizinischer Fragestellungen im Speziellen nicht verheimlichen. Der erste Versuch im Trikot der URO-NEWS wird anhand des Themenschwerpunkts dieser Ausgabe - dem Harnblasenkarzinom als zweithäufigsten malignen Tumor des Urogenitaltrakts - unternommen. Ein besonderer Tumor, ausgehend von einem besonderen Gewebe in einem besonderen (Hohl-)Organ.

Und was hat das nun mit KISS zu tun? Potenziell viel! Fangen wir mit der Diagnostik an: Wer soll wie untersucht werden? Etwa $60 \%$ aller Harnblasenkarzinome gehen auf die beiden Risikofaktoren Rauchen und berufliche Exposition zurück. Es wäre doch einfach, wenn derartig exponierte Personen einem Früherkennungsprogramm unterzogen würden. Eben solches haben wir aber nicht. Deshalb werden die meisten Diagnosen erst mit Auftreten des Kardinalsymptoms Makrohämaturie gestellt. Und die kommt eben nicht selten zu spät.

\section{Investieren Sie eine Minute!}

Die wichtigsten Ursachen für die Entstehung eines Harnblasenkarzinoms sowie die diagnostischen Maßnahmen und Möglichkeiten der urinbasierten Diagnostik beschreibt in dieser URO-NEWS Gerson Lüdecke aus Gießen (s. S. 30). Die endgültige Diagnose des Harnblasenkarzinoms wird im Rahmen einer transurethralen Resektion der Harnblase mit nachfolgender histopathologischer Untersuchung des resezierten Gewebes gestellt. Sämtliche Aspekte der operativen Therapie des nicht muskel- invasiven und des muskelinvasiven Harnblasenkarzinoms werden von Mark Thalgott und Kollegen aus München in Form eines CME-Artikels dargestellt (s. S. 42).

Nach Diagnose eines nicht muskelinvasiven Harnblasenkarzinoms kann anhand einfach zu erhebender und ebenso einfach zu dokumentierender klinischer Parameter eine Risikoabschätzung für ein Rezidiv und eine Progression vorgenommen werden. Ein wichtiges Hilfsmittel sind dabei die Tabellen der EORTC (European Organisation for Research and Treatment of Cancer). Sie basieren auf einer Analyse von sieben Studien mit mehr als 2.500 Patienten und sind seit 2006 verfügbar. Man muss diese eine Minute zum Lesen nur investieren.

Aus dem individuellen Risikoprofil des Betroffenen ergeben sich dann Notwendigkeit und Art einer Metaphylaxe fast wie von selbst. Gerson Lüdecke stellt diese Aspekte auch vor dem Hintergrund neuer Therapieentwicklungen in seinem Beitrag ab Seite 34 komprimiert dar.

Auch die Nachsorge wird auf das individuelle Risikoprofil des Betroffenen bezogen. Die Urethrozystoskopie bleibt dabei der Goldstandard. Die Intervalle können aber modifiziert und der Stellenwert der Urinzytologie und der urinbasierten Marker präzisiert werden (s. S. 38).

Mit vergleichsweise wenigen Informationen könn(t)en wir Urologen unsere Patienten mit einem Harnblasenkarzinom also risikoprofiladaptiert diagnostizieren, therapieren und sogar nachsorgen. KISS eben.

Eine informative Lektüre wünscht Ihnen,

Ihr

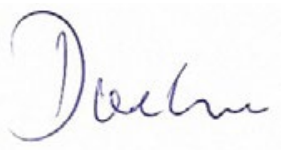

